\title{
A Theoretical Foundation for Software Engineering: A Model Calculus
}

\author{
Dewayne E. Perry \\ Advanced Research in Software Engineering (ARiSE) \\ Electrical and Computer Engineering \\ The University of Texas at Austin \\ Austin, TX, USA \\ perry@ece.utexas.edu
}

\begin{abstract}
Theory is a critical and undervalued part of software engineering and software engineering research. While empirical evaluation is important in both software engineering and software engineering research, there is still a lack of maturity and deep understanding of this critical aspect of both enterprises. The purpose of my unifying theoretical foundation for software engineering is, in part, to illuminate the place and importance of both theory and empirical evaluation. The focus here is on the model calculus and its use in the composition of more complex models to emphasize 1) the taxonomic space of both theory and empirical evaluation, and 2) the complexity resulting from various model compositions. The latter should not be a surprise as the complexity of model compositions merely reflects the fundamental and essential characteristic of our software systems - that is, complexity.
\end{abstract}

Index Terms-Theories and Models of Software Engineering, Model Calculus, Model Composition

\section{INTRODUCTION}

My theory about software engineering [1] (which I claim provides a unifying foundation) comes from my experience as a practicing software engineer and as a software engineering researcher.

Software Engineering consists of two logical parts: design and empirical evaluation (both terms used in their broadest senses). I propose two simple theories, $\mathrm{D}$ and $\mathrm{E}$ as the basis for laying out a unified theoretical foundation for software engineering and software engineering research. I propose theory $\mathrm{D}$ as the theoretical basis for the design part, and theory $\mathrm{E}$ as the theoretical basis for the empirical evaluation part. I then define models for $\mathrm{D}$ and $\mathrm{E}$, using a model calculus, and then compose theories $\mathrm{D}$ and $\mathrm{E}$ in various ways and use the composed models to explore various ways of thinking about the underlying foundations for software engineering and software engineering research.

\section{A. Theories and Models}

The terms "theory" and "model" are used and misused in a variety of ways, often informally and interchangeably. I want to use them in a very specific way: a theory (a more or less abstract entity) is reified, represented, satisfied, etc. by a model (a concrete entity).
This view of theories and models is derived in part from Turski and Maibaum [5] where they state "A specification is rather like a natural science theory of the application domain, but seen as a theory of the corresponding program it enjoys an unmatched status: it is truly a postulative theory, the program is nothing more than an exact embodiment of the specification". I note, however, that I want a theory to be broader than a specification and, more than likely, less formal.

We often use models as a representation of a theory. In natural sciences, the model is often a set of mathematical formulas. In logic, a model is an interpretation of a theory and has certain logical properties. Here again, I want to broaden the notion of a model to be a representation (indeed, a reification) of the theory, and like a theory and model in the logical sense, there can be multiple models that are interpretations or representations of the theory. The model is of paramount importance in design disciplines as it is the visible manifestation of the theory. Of fundamental importance is the fact that a theory can have an arbitrary number of models.

\section{B. More About Theories}

My claim is that the key to a unifying, and a rigorous and systematic, foundation for software engineering, software engineering research, and empirical studies in software engineering and software engineering research is to be found in a focus on theory.

So what is it that I consider to be important in theories: the source of the theories; the structure of the theories; and the use of the theories.

1) Source Of Theories: In terms of sources of theories relevant to software engineering, three different types of theories are important:

1. Scientific theory - Scientific theory is based on observations of the world. They change on the basis of new observations, or new interpretations of observations.

2. Legal theory - Legal theory is quite different: it is based on decisions about the world, and is changed on the basis of new decisions or new interpretations of decisions.

3. Normative theory - Normative theory is different yet and is based on a system of philosophical tenets about what is 
good and bad, and judgments are changed on the basis of new inferences from those tenets or new interpretations of them.

Theories in design disciplines are a combination of all three of the above. They are based on observations, decisions, and judgments about the world. They change on the basis of new observations, decisions, and judgments or on the basis of new interpretations of those observations, decisions and judgments.

2) Structure Of Theories: Markus and Robey [4] distinguish two different theory structures:

1. Variance - In the case of variance, the theoretical structure is a set of laws about interactions or relationships. For example, given a variation in $\mathrm{A}$, what other units can be linked to A such that they account for the variance in A.

2. Process - In the case of process, the theoretical structure is a temporal ordering of activities, steps, or events.

We find both kinds of theoretical structures in design discipline theories depending on what kind, and at what level, we are theorizing about design issues.

3) Use Of Theories: The taxonomy of uses I describe here is derived from Gregor [3]. I distinguish five distinct uses of theories that may be used also in combinations:

1. Description - A theory is used to describe phenomena in terms of its constructs, properties, and relationships, and the boundaries within which those properties and relationships hold. Descriptions are intended to be complete.

2. Prescription - A theory is used to provide a set of constraints on its constructs, properties, and relationships, and the boundaries within which those properties and relationships hold. Prescriptions are intended to emphasize the crucial aspects of the theory.

3. Explanation - A theory is used to explain how, why and when things happen based on causality and methods of demonstration (that is, argumentation). The intent is to provide deeper understanding and insight into the subject phenomena.

4. Prediction - A theory is used to predict what will happen on the basis of necessary and sufficient conditions for the theorized phenomena. The phenomena will not happen if the necessary conditions are withheld; nor will they happen if the sufficient conditions are withheld.

5. Action - A theory provides principles, techniques, and methods for enabling the desired phenomena (for example, achieving a desired goal, or designing or constructing an artifact).

Depending on the context in software engineering, we make use of theory in all these different ways. Theories, of course, influence their models: the source of a theory will affect its model; the structure of a theory will influence the structure of its model; and, the use of a theory will also influence the structure of its model.

\section{A Model CAlculus}

Since theories as used here are informal entities, their composition is also informal and the resulting integration is done informally.
My theory about models, however, has a more formal definition and a set of rules for the model operators. My theory about models is that we can only truly understand the implications of our theories and compositions of theories by reifying them into models that illustrate the true breadth, depth and complexity of those models and compositions.

\section{A. Models}

A model is a tuple consisting of two sets: a set of objects, and a set of mappings (or more informally, transformations, processes, derivations, etc.) from an object in one set of objects to another object in a (usually, but not necessarily, different) set of objects.

$$
\text { Model }=<\{\mathrm{O}\},\{\mathrm{M}\}>
$$

Models can be arbitrarily considered to be either atomic or open-structured. If viewed as atomic, then their structure is abstract - that is, it is hidden and the model is considered as a whole, indivisible entity. If viewed as open-structured, all the individual objects and mappings are visible in the model.

\section{B. Model Syntax}

The following are the special symbols in the model calculus (in order of precedence):

- "+" a unary operator on objects that indicates 1 or more of the designated objects.

- “:" a binary operator on models and model components that indicates composition of two such elements.

- “*” a binary operator on objects that delineates an object in the Cartesian space of two objects. This can be thought of as functional application of the one object to the other yielding a specific object as its value.

- " $\rightarrow$ " a binary operator that maps one object onto another.

- Parentheses may be used to clarify the use of these operators.

Generally I use one alphabetic letter to denote objects and models, though of course one may use descriptive names for clarity if needed. When it is unambiguous one may elide the use of the composition operator. For example, AB can be used to compose the two elements instead of $\mathrm{A}: \mathrm{B}$. For clarity in distinguishing between models I sometimes use lower case letters for one of the model's elements.

\section{Mappings}

All possible mappings are possible in this model calculus:

- $\quad$ One to one mappings are indicated by A $\rightarrow$ B.

- Many to one mappings are indicated in several different ways. For example, $\mathrm{A} * \mathrm{~B} \rightarrow \mathrm{C}$, and $\mathrm{A}+\rightarrow \mathrm{B}$.

- $\quad$ One to many mappings are indicated by $\mathrm{A} \rightarrow \mathrm{B}+$ and $\mathrm{A} \rightarrow \mathrm{B} * \mathrm{C}$.

- Many to many mappings are indicated by any combinations using "+" and "*" together with " $\rightarrow$ " 


\section{Calculus Rules}

The following are the distribution rules among expressions about various operators.

- ":?" is both left and right distributive over models.

- ":" is left distributive over "+", “*”, and " $\rightarrow$ ".

Examples of the first distribution rule can be seen in the next section. Examples of the second are as follows:

$$
\begin{aligned}
& \mathrm{O}^{1} \rightarrow \mathrm{O}^{2:} \mathrm{AM}=\mathrm{O}^{1}: \mathrm{AM} \rightarrow \mathrm{O}^{2}: \mathrm{AM} \\
& \mathrm{O}^{1}{ }^{*} \mathrm{O}^{2} \rightarrow \mathrm{O}^{3:} \mathrm{AM}=\mathrm{O}^{1}: \mathrm{AM} * \mathrm{O}^{2}: \mathrm{AM} \rightarrow \mathrm{O}^{3}: \mathrm{AM} \\
& \mathrm{O}^{1+} \rightarrow \mathrm{O}^{2:} \mathrm{AM}=\left(\mathrm{O}^{1}: \mathrm{AM}\right)+\rightarrow \mathrm{O}^{2}: \mathrm{A}
\end{aligned}
$$

There is one rule about the operator "+" (which implies that "+" is left distributive) over "*" and " $\rightarrow$ ". For example,

$$
\begin{array}{ll}
(\mathrm{A} \rightarrow \mathrm{B})+ & =\mathrm{A}+\rightarrow \mathrm{B}+ \\
(\mathrm{A} * \mathrm{~B})+ & =\mathrm{A}+* \mathrm{~B}+
\end{array}
$$

\section{E. Model Composition}

Models (and theories) can be composed to yield further models (and theories). As mentioned above, model compositions are indicated by A : B. Further, models can be arbitrarily considered to be atomic (that is, its structure is hidden) or open-structured (that is, the objects and mappings are visible).

For example, composing an open structured model (OSM) with an atomic model (AM) results in a model via left distribution of the atomic model AM over the open-structure model OSM.

$$
\begin{aligned}
\text { OSM } & : A M= \\
& <\{O\},\{M\}>: A M= \\
& <\{O\}: A M,\{M\}: A M>= \\
& <\left\{O^{1}: A M \ldots O^{n}: A M\right\},\left\{M^{1}: A M \ldots M^{n}: A M\right\}>
\end{aligned}
$$

Note that in this case, the above left distribution rules of composition over mapping operators can then be applied.

On the other hand, composing an atomic model with an open structure model yields a number of models (equal to the number of objects and mappings) via right distribution of the atomic model AM over the open-structured model OSM.

$$
\begin{aligned}
& \mathrm{AM}: \mathrm{OSM}= \\
& \text { AM : }<\{\mathrm{O}\},\{\mathrm{M}\}>= \\
& <\mathrm{AM}:\{\mathrm{O}\}, \mathrm{AM}:\{\mathrm{M}\}>= \\
& <\left\{\mathrm{AM}: \mathrm{O}^{1}, \ldots, \mathrm{AM}: \mathrm{O}^{\mathrm{n}}\right\},\left\{\mathrm{AM}: \mathrm{M}^{1}, \ldots, \mathrm{AM}: \mathrm{M}^{\mathrm{n}}\right\}>= \\
& \text { AM:O }{ }^{1}, \ldots, A M: O^{n}, A M: M^{1}, \ldots, A M: M^{n}
\end{aligned}
$$

Each of these compositions is a model restricted to that particular object or mapping. Note that because of the left distributive rule of composition over mapping operators, nothing further can be done if AM remains atomic. However, if AM is treated instead as an open-structure model, then the various objects and mappings can be left distributed over that now open model.

\section{RECAP OF MODELS D AND E}

The set the context of the subsequent discussion, the following are the models of D and E from [1].

\section{A. Model of $D$}

The model of D consists of three elements (objects) and six transformations (mappings, or, if you will, processes). The elements are as follows:
- $\quad$ W - The world, but more specifically, the part of the world relevant to the theory

- $\quad \mathbf{T}$ - The theory initiated by observations and abstractions

- $\quad \mathbf{M}-$ A model that reifies, represents or satisfies the theory $\mathrm{T}$

The mappings involving these elements of the model are as follows:

- $\quad \mathbf{W} \rightarrow \mathbf{T}-$ Generate a theory: observe and abstract from the world $\mathrm{W}$ to create a theory $\mathrm{T}$

- $\mathbf{T} \rightarrow \mathbf{M}-$ From the theory $\mathrm{T}$ create/evolve a model $\mathrm{M}$

- $\quad \mathbf{T} \rightarrow \mathbf{T}-$ Evolve theory $\mathrm{T}$ until satisfied

- $\quad \mathbf{M} \rightarrow \mathbf{M}-$ Evolve the model $\mathrm{M}$ until satisfied

- $\quad \mathbf{M} \rightarrow \mathbf{T}-$ Change the theory $\mathrm{T}$ to better conform to model $\mathrm{M}$

- $\mathbf{M} * \mathbf{W} \rightarrow \mathbf{W}$ - Inject model $\mathrm{M}$ into the world $\mathrm{W}$ thereby changing it (which depends on both the model and the world before the injection of the model into it).

\section{B. Model of $E$}

The basic elements in the model and their interrelationships are: theory $\mathbf{T}$, hypothesis $\mathbf{H}$, and evaluation $\mathbf{E}$.

The following transformations represent the processes of conducting an empirical study.

- $\mathbf{T} \rightarrow \mathbf{H}-$ derive an hypothesis $\mathrm{H}$ from theory $\mathrm{T}$

- $\quad \mathbf{H} \rightarrow \mathbf{E}-$ create an appropriate evaluation based on $\mathrm{H}$

- $\mathbf{E} * \mathbf{T} \rightarrow \mathbf{T}-$ reconcile theory and reality - i.e., on the basis of the evaluation and the current theory $\mathrm{T}$, revise T.

\section{Taxonomic Structure of the Compositions with $D \& E$}

A taxonomic space of a composition is the resulting matrix of composing two models and considering both models to be open-structured. For example, E:D yields a set of 16 models and a taxonomic space 6 ( 3 objects and 3 mappings) x 9 (3 objects and 6 mappings) $=54$ evaluations of objects and mappings. D:D yields a set of 19 models and a taxonomic space of $9 \times 9=81$ combinations of objects and mappings. Evaluating D:D, (E:(D:D)) yields a set of 27 models and a taxonomic space of $6 \times 9 \times 9=496$ evaluations of objects and mappings. It is clear that the more complex the models are, the larger the taxonomic space.

\section{Models of Software Engineering and Software Engineering Research}

The claim I make in [1] is that D is a model of the design part of software engineering - that is, creating the requirements for the system to be implemented, and implementing a system that satisfies those requirements.

Further, E:D is a model of the empirical evaluation part of software engineering - that is, the measurement and evaluation part such as reviews, and the various forms of analysis and testing.

$\mathrm{D}: \mathrm{D}$ is then a model for the design part of software engineering research and E:(D:D) is the evaluation part of software engineering research.

See [1] for the full discussion of these compositions 


\section{An Alternative EMPIRICAL MODEL E'}

In [1], the theory and model of empirical evaluation was exceedingly simple - useful for the purposes of illustrating my approach about its centrality and utility in evaluating the design parts of software engineering and software engineering research. Here i propose a more realistic model of empirical evaluations, one that more realistically reflects empirical science.

What is missing in $\mathrm{E}$ is the centrality of instruments as a critically important [2] element in empirical science. They are the lens through which we observe the world. To paraphrase Wittgenstein [6], the limits of my instruments are the limits of my world. They enhance, limit, and color our view of the world. In natural sciences, instruments are often physical creations; in behavioral sciences they are often intellectual creations. Humans are common instruments in both. Instruments may be active or passive. They may be theoryladen or transparent and neutral. They may be reliable and standardized or not. In any case, they are a critical part of the empirical apparatus and as such will play a critical part in any empirical evaluation endeavor.

We remedy this problem in E'.

\section{A. Theory E'}

In Figure 1, we illustrate an expanded version of empirical evaluations showing the position of instruments in empirical endeavors.

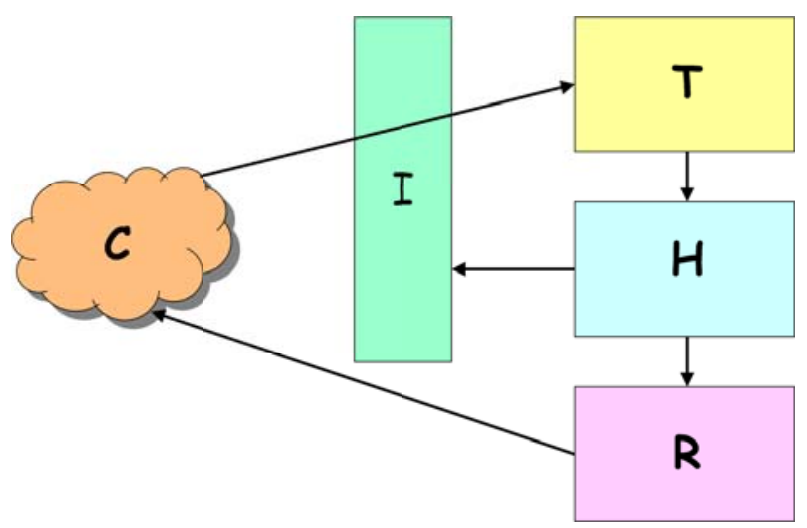

Figure 1-Basic Empirical Science

Theory E' then is described as follows:

- Given a theory, generate an hypothesis to test some part of the theory

- From the hypothesis, generate a regimen (that is, a treatment) and one or more instruments.

- The regimen is a treatment mechanism to generate desired data in the designated context.

- The instruments are used to provide observations about the generated data resulting from the manipulations of the independent variables.

- On the basis of the instrumented observations, generate a set of result analyses to use to reconcile the observations with the theory, revising the theory if needed.
Iteration is inherent in $\mathrm{E}^{\prime}$ as it is in both $\mathrm{E}$ and $\mathrm{D}$. We iterate revising $\mathrm{T}$ and generating new hypotheses, regimens and instruments to refine theory T. Theory E' then provides us with a more realistic view of empirical ventures.

\section{B. Model E'}

Of course, with the richer theory E', we need a richer model to represent E'. The model of E' has 8 objects and 7 mappings. The objects are as follows:

- $\mathrm{T}$ - Theory

- $\mathrm{H}$ - Hypothesis

- $\mathrm{R}$ - Regimen (Treatment) manipulates independent variables in context $\mathrm{C}$

- $\quad \mathrm{C}$ - Context (where the independent and dependent variables are found) in which $\mathrm{R}$ is applied and observed by I

- D - Data resulting from application of the regimen

- I - Instrument

- $\mathrm{O}$ - Observations of the data $\mathrm{D}$ seen through instruments I

- A - Analyses based on the observations

The mappings are as follows:

- $\mathrm{T} \rightarrow \mathrm{H}-$ deriving a hypothesis $\mathrm{H}$ from theory $\mathrm{T}$

- $\mathrm{H} \rightarrow \mathrm{I}$ - create appropriate instruments/mechanisms for observing the dependent variables

- $\quad \mathrm{H} \rightarrow \mathrm{R}$ - create appropriate regimen with manipulations of independent variables

- $\quad \mathrm{R}^{*} \mathrm{C} \rightarrow \mathrm{D}$ - performing the experiment: apply regimen to context yielding resulting data

- $\quad \mathrm{I} * \mathrm{D} \rightarrow \mathrm{O}$ - use instrument I to provide observations of data $\mathrm{D}$

- $\mathrm{O} \rightarrow \mathrm{A}-$ generate analyses from the observations $\mathrm{O}-$ i.e., drawing conclusions from the experiment

- $\quad \mathrm{A}^{*} \mathrm{~T} \rightarrow \mathrm{T}^{\prime}-$ reconcile theory and reality: evaluate $\mathrm{T}$ possibly revising it into T'

\section{Evaluating Empirical Studies}

With the more complex theory and model of E' comes a larger number of models as a results of the composition E':E' 16 for E':E' instead of 9 for E:E. The taxonomic space is significantly larger as well - 225 elements instead of 64.almost a factor of 4 larger. The usefulness of the more complete theory and model is the exposure of what in many cases are critically important issues.

1) Using an Atomic E' To Evaluate an Open-Structured e': In this and the following sections we use E' and e' to distinguish the two uses of $E$ '. In this and the next section, we also illustrate the usefulness in the atomic versus openstructured. In this case we consider E' to be atomic, and used as a whole to evaluate the individual objects and mappings of e'.

- E':t - evaluate the theory of e'

- $E^{\prime}: h$ - evaluate the hypothesis of e'

- E':I - evaluate the instrument of e'

- $\quad E^{\prime}: r$ - evaluate the regimen of e'

- $\quad E^{\prime}: c$ - evaluate the context of e' 
- $\quad E^{\prime}: d$ - evaluate the data of e'

- E':o - evaluate the observations of e'

- $\quad E^{\prime}: a-$ evaluate the analyses used in e'

- $E^{\prime}:(t \rightarrow h)-$ evaluate the derivation of the hypothesis of e' from the theory of e'

- $E^{\prime}:(h \rightarrow i)-$ evaluate the determination of the instrument for e' from the hypothesis of e'

- $\quad E^{\prime}:(h \rightarrow r)-$ evaluate the design of the regimen derived from the hypothesis for e'

- $\quad E^{\prime}:\left(r^{*} c \rightarrow d\right)$ - evaluate generation of data by means of the application of the regimen for evaluating e' to its context

- $\quad E^{\prime}:\left(i^{*} d \rightarrow 0\right)-$ evaluate the generation of observations from the data using the instruments of $e^{\text {' }}$

- $E^{\prime}:(0 \rightarrow a)$ - evaluate the derivations of the analysis from the observations for e'

- $E^{\prime}:\left(a^{*} t \rightarrow t^{\prime}\right)$ - evaluate the reconciliation of the analysis and the theory and possibly revising the theory for e'

The interpretation of elements of the model E':e' may be as follows. E':t may evaluate theory completeness and consistency, or theory appropriateness and adequacy. $E^{\prime}: h$ may evaluate the appropriateness of the hypothesis $h$ relative to the theory $t$, or evaluate the construct validity of hypothesis $h$. E':r may consider the appropriateness of regimen $r$ relative to hypothesis $h$ and theory $t$, or consider the construct and/or internal validity of regimen $\mathrm{r}$. E':c may evaluate the appropriateness of context $\mathrm{c}$ relative to theory $\mathrm{t}$ and hypothesis $h$. $E^{\prime}:(t \rightarrow h)$ may evaluate the quality of the hypothesis generation process, or evaluate construct validity. $E^{\prime}:(h \rightarrow r)$ may address the problems of construct and internal validity.

2) Using an Open-Structured E' To Evaluate Atomic e': In this case, we consider E' to be open-structured and e' to be atomic, thus evaluating e' as a whole in terms of the individual elements of E'

The set of objects then in the composition of E':e' are as follows.

- $\mathrm{T}: \mathrm{e}^{\prime}-\mathrm{a}$ theory about evaluating e'

- H:e' - an hypothesis about evaluating e'

- R:e' - a regimen for evaluating e'

- C:e' - a context for evaluating e'

- D:e' - data for e' resulting from the application of the regimen for evaluating e'

- I:e' - instruments for evaluating e'

- O:e' - observations of the data seen through the instruments for evaluating $\mathrm{e}^{\prime}$

- A:e' - analyses based on the observations in evaluating e'

The set of mappings in the composition of E':e' are as follows:

- $(\mathrm{T} \rightarrow \mathrm{H}): \mathrm{e}^{\prime} \quad=\mathrm{T}: \mathrm{e}^{\prime} \rightarrow \mathrm{H}: \mathrm{e}^{\prime}$

Deriving an hypothesis for evaluating e' from the theory about evaluating e'

- $(\mathrm{H} \rightarrow \mathrm{I}): \mathrm{e}^{\prime} \quad=\mathrm{H}: \mathrm{e}^{\prime} \rightarrow \mathrm{I}: \mathrm{e}^{\prime}$
Determining an instrument to be used in the evaluation of e' from the hypothesis for evaluating e'

- $(H \rightarrow R): e^{\prime} \quad=H: e^{\prime} \rightarrow$ R:e'

Determining a regimen for evaluating e' from the hypothesis for evaluating e'

- $\quad\left(\mathrm{R}^{*} \mathrm{C} \rightarrow \mathrm{D}\right): \mathrm{e}^{\prime} \quad=\mathrm{R}: \mathrm{e}^{*} \mathrm{C}^{*} \mathrm{e}^{\prime} \rightarrow \mathrm{D}: \mathrm{e}^{\prime}$

Performing the evaluation of e' in the context for evaluating e' yielding the evaluation data for e'

- $\left(\mathrm{I}^{*} \mathrm{D} \rightarrow \mathrm{O}\right): \mathrm{e}^{\prime} \quad=\mathrm{I}: \mathrm{e}^{\prime *} \mathrm{D}: \mathrm{e}^{\prime} \rightarrow \mathrm{O}: \mathrm{e}^{\prime}$

Applying the instrument for evaluating e' to the evaluation data for e', yielding evaluation observations about e'

- $(\mathrm{O} \rightarrow \mathrm{A}): \mathrm{e}^{\prime} \quad=\mathrm{O}: \mathrm{e}^{\prime} \rightarrow \mathrm{A}: \mathrm{e}^{\prime}$

Deriving an analysis of the evaluation of e from the evaluation observations

- $\left(\mathrm{A}: \mathrm{T} \rightarrow \mathrm{T}^{\prime}\right): \mathrm{e}^{\prime} \quad=\mathrm{A}: \mathrm{e}^{\prime} * \mathrm{~T}: \mathrm{e}^{\prime} \rightarrow \mathrm{T}^{\prime}: \mathrm{e}^{\prime}$

Reconciling the evaluation of e' with the theory about evaluating e' and possibly revising that theory.

3) Expanding the Details - Both Models Open-Structured: To understand fully the implication of what is hidden by keeping one of the models atomic (which does provide a useful abstraction in reducing the inherent complexity of model composition), we consider an example from each of the preceding compositions to illustrate that complexity.

Let us consider a simple example from using E' to evaluate an object of e', E':t. We have a set of objects in this model as follows:

- $\mathrm{T}: \mathrm{t}-\mathrm{a}$ theory $\mathrm{T}$ about the theory $\mathrm{t}$ of $\mathrm{e}$ '

- H:t - an hypotheses $\mathrm{H}$ about the theory $t$ of $\mathrm{e}^{\text {' }}$

- I:t - an instrument I used for theory $t$ of e'

- R:t - a regimen $\mathrm{R}$ used for the theory $t$ of $\mathrm{e}^{\prime}$

- C:t - a context $\mathrm{C}$ appropriate for theory $t$ of $e^{\text {' }}$

- D:t - data for the theory t of e'

- O:t - observations for the theory $t$ of e'

- A:t - analysis relevent to the theory t of e'

We then use these objects in the mappings of E' for the evaluation of $\mathrm{t}$.

- $(\mathrm{T} \rightarrow \mathrm{H}): \mathrm{t} \quad=\mathrm{T}: \mathrm{t} \rightarrow \mathrm{H}: \mathrm{t}$

Generating an hypothesis $\mathrm{H}$ about $\mathrm{t}$ from the theory $\mathrm{T}$ about $\mathrm{t}$

- $(\mathrm{H} \rightarrow \mathrm{I}): \mathrm{t} \quad=\mathrm{H}: \mathrm{t} \rightarrow \mathrm{I}: \mathrm{t}$

Deriving an instrument $\mathrm{I}$ for $\mathrm{t}$ from the hypothesis $\mathrm{H}$ about $\mathrm{t}$

- $(\mathrm{H} \rightarrow \mathrm{R}): \mathrm{t} \quad=\mathrm{H}: \mathrm{t} \rightarrow \mathrm{R}: \mathrm{t}$

Deriving a regimen $\mathrm{R}$ about $\mathrm{t}$ from the hypothesis $\mathrm{H}$ about $\mathrm{t}$

- $\left(\mathrm{R}^{*} \mathrm{C} \rightarrow \mathrm{D}\right): \mathrm{t} \quad=\mathrm{R}: \mathrm{t}^{*} \mathrm{C}: \mathrm{t} \rightarrow \mathrm{D}: \mathrm{t}$

Applying the regimen for $t$ to the context of $t$ to generate the data about $t$

- $(\mathrm{I} * \mathrm{D} \rightarrow \mathrm{O}): \mathrm{t} \quad=\mathrm{I}: \mathrm{t} * \mathrm{D}: \mathrm{t} \rightarrow \mathrm{O}: \mathrm{t}$ Using the instruments for $t$ to yield observations about $t$ from the data of $t$

- $(\mathrm{O} \rightarrow \mathrm{A}): \mathrm{t} \quad=\mathrm{O}: \mathrm{t} \rightarrow \mathrm{A}: \mathrm{t}$

Deriving analyses about $t$ from the observations on $t$

- $\left(\mathrm{A}^{*} \mathrm{~T} \rightarrow \mathrm{T}^{\prime}\right): \mathrm{t} \quad=\mathrm{A}: \mathrm{t}^{*} \mathrm{~T}: \mathrm{t} \rightarrow \mathrm{T}^{\prime}: \mathrm{t}$ 
Reconciling the analyses about $t$ with the theory about $t$, possibly modifying the theory about $t$

In the second example where we applied individual objects and mappings to e' as a whole (that is, as atomic), to fully understand the implications of that we must open the structure of e'. Consider the example, $(\mathrm{T} \rightarrow \mathrm{H}): \mathrm{e}^{\prime}=\mathrm{T}: \mathrm{e}^{\prime} \rightarrow \mathrm{H}: \mathrm{e}^{\prime}$.

$$
\text { - }(\mathrm{T} \rightarrow \mathrm{H}): \mathrm{t} \quad=\mathrm{T}: \mathrm{t} \rightarrow \mathrm{H}: \mathrm{t}
$$

Generating an hypothesis $\mathrm{H}$ about theory $t$ from the theory $\mathrm{T}$ about theory $t$. The rest of the compositions of objects are analogous

$$
\begin{array}{ll}
\text { - } \quad(\mathrm{T} \rightarrow \mathrm{H}): \mathrm{h} & =\mathrm{T}: \mathrm{h} \rightarrow \mathrm{H}: \mathrm{h} \\
\text { - } \quad(\mathrm{T} \rightarrow \mathrm{T} \rightarrow \mathrm{H}): \mathrm{I} & =\mathrm{T}: \mathrm{i} \rightarrow \mathrm{H}: \mathrm{i} \\
\text { - } \quad(\mathrm{T} \rightarrow \mathrm{H}): \mathrm{c} & =\mathrm{T}: \mathrm{r} \rightarrow \mathrm{H}: \mathrm{r} \\
\text { - } \quad(\mathrm{T} \rightarrow \mathrm{H}): \mathrm{d} & =\mathrm{T}: \mathrm{d} \rightarrow \mathrm{H}: \mathrm{H}: \mathrm{d} \\
\text { - } \quad(\mathrm{T} \rightarrow \mathrm{H}): \mathrm{o} & =\mathrm{T}: \mathrm{o} \rightarrow \mathrm{H}: \mathrm{o} \\
\text { - } \quad(\mathrm{T} \rightarrow \mathrm{H}): \mathrm{a} & =\mathrm{T}: \mathrm{a} \rightarrow \mathrm{H}: \mathrm{a} \\
\text { - } \quad(\mathrm{T} \rightarrow \mathrm{H}):(\mathrm{t} \rightarrow \mathrm{h}) & =\mathrm{T}:(\mathrm{t} \rightarrow \mathrm{h}) \rightarrow \mathrm{H}:(\mathrm{t} \rightarrow \mathrm{h})
\end{array}
$$

Generating an hypothesis $\mathrm{H}$ about the process of generating an hypothesis $h$ from the theory $t$ from the theory $\mathrm{T}$ about generating the hypothesis $h$ from $t$. The rest of the compositions of mappings of this form are analogous.

- $(\mathrm{T} \rightarrow \mathrm{H}):(\mathrm{h} \rightarrow \mathrm{i}) \quad=\mathrm{T}:(\mathrm{h} \rightarrow \mathrm{i}) \rightarrow \mathrm{H}:(\mathrm{h} \rightarrow \mathrm{i})$

- $\quad(\mathrm{T} \rightarrow \mathrm{H}):(\mathrm{h} \rightarrow \mathrm{r}) \quad=\mathrm{T}:(\mathrm{h} \rightarrow \mathrm{r}) \rightarrow \mathrm{H}:(\mathrm{h} \rightarrow \mathrm{r})$

- $\quad(\mathrm{T} \rightarrow \mathrm{H}):\left(\mathrm{r}^{*} \mathrm{c} \rightarrow \mathrm{d}\right)=\mathrm{T}:\left(\mathrm{r}^{*} \mathrm{c} \rightarrow \mathrm{d}\right) \rightarrow \mathrm{H}:\left(\mathrm{r}^{*} \mathrm{c} \rightarrow \mathrm{d}\right)$

Generating an hypothesis $\mathrm{H}$ about the process of generating data by the application of the regimen $r$ to the context $c$ from the theory $\mathrm{T}$ about the process of generating data by the application of the regimen $r$ to the context $c$. The remaining mappings of this form are also analogous to this one.

- $\quad(\mathrm{T} \rightarrow \mathrm{H}):\left(\mathrm{i}^{*} \mathrm{~d} \rightarrow \mathrm{o}\right)=\mathrm{T}:\left(\mathrm{i}^{*} \mathrm{~d} \rightarrow \mathrm{o}\right) \rightarrow \mathrm{H}:\left(\mathrm{i}^{*} \mathrm{~d} \rightarrow \mathrm{o}\right)$

- $(\mathrm{T} \rightarrow \mathrm{H}):(\mathrm{o} \rightarrow \mathrm{a}) \quad=\mathrm{T}:(\mathrm{o} \rightarrow \mathrm{a}) \rightarrow \mathrm{H}:(\mathrm{o} \rightarrow \mathrm{a})$

- $\quad(\mathrm{T} \rightarrow \mathrm{H}):\left(\mathrm{a}^{*} \mathrm{t} \rightarrow \mathrm{t}^{\prime}\right)=\mathrm{T}:\left(\mathrm{a}^{*} \mathrm{t} \rightarrow \mathrm{t}^{\prime}\right) \rightarrow \mathrm{H}:\left(\mathrm{a}^{*} \mathrm{t} \rightarrow \mathrm{t}^{\prime}\right)$

These two examples delineating the full composition of several instances of the composition E':e' provide a good illustration of the size of the full taxonomic matrix resulting from such a composition.

\section{Discussion of E'}

Theory and model E' provide a more detailed and fuller approach to empirical evaluation. The difference between $\mathrm{E}$ and E' is primarily that of detail with E' providing an emphasis on the importance of instruments, observations, and analyses. Both E and E' are intended to cover the entire range of empirical studies from exploratory through to rigorous experimental studies. The theory $\mathrm{T}$ may be vague and ill formed when doing exploratory work, or well-formed and mature when doing explanatory work (typically using a null hypothesis experiment). The hypothesis $\mathrm{H}$ may be generic and open-ended when doing exploratory work, or focused and specific when doing explanatory work. The instruments I and regimens $\mathrm{R}$ (i.e., treatments) may be human and opportunistic when doing exploratory work, or specifically and well- designed in both cases when doing explanatory work. Reconciliation of theory and reality may result in theory generation when doing exploratory work, or adding support for the theory or revising it when doing explanatory work.

With respect to the issues of validity, the various elements of E':e' are as follows:

- Construct validity - the most critical issue for technological and design decisions and addressed by at least $E^{\prime}: h, E^{\prime}: i$, and $E^{\prime}:(h \rightarrow i)$

- Internal validity - is addressed by at least $\mathrm{E}^{\prime}: \mathrm{h}, \mathrm{E}^{\prime}: \mathrm{i}$, $E^{\prime}: r, E^{\prime}:(h \rightarrow r)$, and $E^{\prime}:\left(r^{*} c \rightarrow d\right)$

- $\quad$ Statistical validity - is addressed by at least $E^{\prime}: a$, $\mathrm{E}^{\prime}:(\mathrm{o} \rightarrow \mathrm{a})$, and $\mathrm{E}^{\prime}:\left(\mathrm{a} * \mathrm{t} \rightarrow \mathrm{t}^{\prime}\right)$

- External validity - is addressed by at least $\mathrm{E}^{\prime}: \mathrm{c}$

An interesting question is whether the E' should be used to evaluate itself or whether some other theory and model, perhaps simpler, should be used instead - for example E. As seen above the taxonomic space is large using $\mathrm{E}$ ' to evaluate itself. This is not necessarily a bad thing as it does have the utility of illustrating just how large and complex the problem of evaluating our evaluations is. The simpler theory E hides a large amount of that complexity. Further exploration of this issue is needed to understand the utility, strengths and weaknesses of alternative approaches.

\section{An Alternative Design Model D’}

In [1], the theory and model of design $\mathrm{D}$ was a generic and simple model of iterative design. Indeed, there is a wide variety of more detailed theories and models that could be (and have been) proposed and are in use ranging from step-wise refinement to Boehm's spiral model of development. We use different techniques and methods to guide and direct the process creating theories, generating models from theories, specifying what theories and models look like, analyzing them, etc.

An example of such a more detailed theory and model is that of Nuseibeh, Kramer and Finkelstein's multiple viewpoints [7] approach in which there are different stakeholders with respect to the problem to be solved; these stakeholders have different views on what is important in the theory (i.e., requirements that need to be captured; and eventually any and all apparent and real conflicts need to be resolved to provide a consistent theory (i.e., a consistent set of requirements).

\section{A. Theory D'}

The added details to theory $D^{\prime}$ are primarily in the generation of a theory that is then the basis for creating the corresponding model.

- We as multiple stakeholders observe and abstract some specific part of the world and create a part of a theory that is relevant to us

- These stakeholders' partial theories must be merged into a consistent single theory.

- From that theory we create a usable model to reify or represent that theory.

- We iteratively adjust both the theory (and sometimes the stakeholders' partial theories) and the model as our 
understanding of the theory and its model evolves, both iteratively and interactively.

- When satisfied that the model adequately represents the theory, we inject the model into the world.

- Injecting the model into the world changes the world.

- The changes brought about by these changes as well as other changes often lead to adjustments and extensions to the stakeholders partial theories and the merged theory.

- Changes to the theory in turn lead to further changes in the model and the world.

So, theory iteration becomes more complex and in turn makes the iteration between the theory and model more complex as well.

\section{B. Model D'}

For the most part, the theory of D is carried over into D'. As noted in the theory of $\mathrm{D}^{\prime}$ it is the structure of deriving the theory $\mathrm{T}$ that is changed and provided in more detail

- $\quad \mathbf{W}$ - The world, but more specifically, the part of the world relevant to the theory

- $\mathbf{S}$ - The stakeholders who create partial theories

- $\quad$ - Partial theories

- $\mathbf{T}-$ The theory resulting from merging partial theories

- $\quad \mathbf{M}-$ A model that reifies, represents or satisfies the theory $\mathrm{T}$

The mappings involving these elements of the model are as follows:

- $\mathbf{S}+* \mathbf{W} \rightarrow \mathbf{P}+-$ Stakeholders generate partial theories: observe and abstract from the world $\mathrm{W}$ to create a partial theories $\mathrm{P}+$

- $\quad \mathbf{P}+\rightarrow \mathbf{T}-$ The partial theories are merged into a consistent theory

- $\quad \mathbf{T} \rightarrow \mathbf{P}+-$ the partial are adjusted due to consistency issues in theory $\mathrm{T}$

- $\quad \mathbf{T} \rightarrow \mathbf{T}-$ Evolve theory $\mathrm{T}$ until satisfied

- $\mathbf{T} \rightarrow \mathbf{M}-$ From the theory $\mathrm{T}$ create/evolve a model $\mathrm{M}$

- $\quad \mathbf{M} \rightarrow \mathbf{M}-$ Evolve the model $\mathrm{M}$ until satisfied

- $\quad \mathbf{M} \rightarrow \mathbf{T}-$ Change the theory $\mathrm{T}$ to better conform to model $\mathrm{M}$

- $\quad \mathbf{M} * \mathbf{W} \rightarrow \mathbf{W}$ - Inject model $\mathrm{M}$ into the world $\mathrm{W}$ thereby changing it (which depends on both the model and the world before the injection of the model into it).

A possible alternative to $\mathrm{T} \rightarrow \mathrm{P}+$ to capture the process of theory consistency issues resulting from either inconsistencies in the partial theories $\mathrm{P}+$ or changes in the theory $\mathrm{T}$ to better to conform to the model $\mathrm{M}$ is to use instead a much more complex mapping: $\mathrm{T} \rightarrow(\mathrm{S}+* \mathrm{~W} \rightarrow \mathrm{P}+)$. This mapping would represent the process of the theory $\mathrm{T}$ generating a new set of partial theories by the various stakeholders. In $\mathrm{T} \rightarrow \mathrm{P}+$, the need for the stakeholders to modify their partial theories is implicit rather than explicit.

As always, then, how models reify their theories is a creative process often with various alternatives of perhaps differing utility and clarity. And, there is usually a tradeoff between simplicity and expressiveness.

\section{Compositions with D'}

Analogous to D:D in [1], the obvious thing to do is to use D' in the same way to create a theory and model about creating a theory and model. The details of the composition of D':D'. I leave as an exercise to the reader to help expand their understanding of the model calculus in the filling out of the taxonomic matrix.

The interesting question here is whether D' is appropriate since a theory is created from partial theories created by various stakeholders. There are two cases to consider: where there is one researcher, and where there are more than one researcher. D' actually can be used to represent both of these cases as the "+" operation is defined to mean "one of more" stakeholders. As we often work in teams, even as researchers, $\mathrm{D}^{\prime}$ is quite appropriate for the task.

The question, however, of whether this is the best solution, or whether a different, or simpler, theory and model is more useful or appropriate. This, as with the same question about evaluating evaluations needs further study.

\section{SUMMARY}

The motivation for my research is to create a unifying theoretical basis for software engineering and software engineering research. In particular I want to make two important points: 1) the usefulness of my view of theories and models, and 2) the important distinction between design and evaluation, both of which are critical parts of software engineering and software engineering research.

In making this distinction I especially want to emphasize the centrality and criticality of theory in both software engineering and software engineering research. Too often little is explicit in the underlying theoretical basis of our work in software engineering and software engineering research. We need to make our underpinning theories more explicit and central in our work.

Similarly, I want to emphasize the centrality and criticality of empirical evaluations in both enterprises as well. Too little attention is paid to the evaluation part of software engineering and software engineering research with the primary, and sometimes sole, emphasis put on design (obviously critically important in its own right). We need a more explicit, systematic, and deeper approach to empirical evaluations in both software engineering and software engineering research.

My goal has been to illustrate the very rich space that we can define using small and relatively simple theories - in this case making D' and E' slightly richer than D and E. The beauty of this approach is that the composition of these theories and their models extend our understanding and illuminate the taxonomic space for the resulting theories and their models.

Finally, there are two useful properties of my approach: 1) regularity among the various theories; and 2) the utility of the two levels of abstraction (atomic and open-structured) that I use to provide an intuitive high level abstraction, and the explicit low level details. 


\section{ACKNOWLEDGMENT}

This research was sponsored in part by NSF CISE SRS Grant CCF-0820251.

\section{REFERENCES}

[1] Dewayne E Perry. "A Unifying Theoretical Foundation for Software Engineering", Conference on Software Engineering and Data Engineering (SEDE), Las Vegas, June 2011, pp 1-6. http://users.ece.utexas.edu/ perry/work/papers/DP-sede11.pdf

[2] David Gooding, Trevor Pinch, and Simon Schaffer, Editors. The Uses of Experiment: Studies in the Natural Sciences. Cambridge: Cambridge University Press, 1989.

[3] Shirley Gregor. "The Nature of Theory in Information Systems", MIS Quarterly 30 (2006), pp 611-642
[4] M. L. Markus and D Robey. "Information Technology and Organizational Change: Causal Structure in theory and Research", Management Science 34:5 (1988), pp 583-598

[5] Turski, Wladyslaw M. and Maibaum, Thomas S. E. The Specification of Computer Programs. Reading, Mass: AddisonWesley, 1987.

[6] Wittgenstein, Ludwig. Tractatus Logico-Philosophicus, ed. A. J. Ayer, London: Routledge \& Kegan Paul, 1961. Section 5.6

[7] Bashar Nuseibeh, Jeff Kramer, Anthony Finkelstein. Expressing the Relationships Between Multiple Views in Requirements Specification, ICSE, 1993. 